\title{
Optimized regulator for the quantized anharmonic oscillator
}

\author{
J. Kovacs, * S. Nagy ${ }^{\dagger}$ and K. Sailer \\ Department of Theoretical Physics, University of Debrecen, \\ P.O. Box 5, H-4010 Debrecen, Hungary \\ *jkovacs@phys.unideb.hu \\ †nagys@dtp.atomki.hu \\ ‡sailer@phys.unideb.hu
}

Received 22 January 2015

Accepted 9 February 2015

Published 14 April 2015

\begin{abstract}
The energy gap between the first excited state and the ground state is calculated for the quantized anharmonic oscillator in the framework of the functional renormalization group method. The compactly supported smooth regulator is used which includes various types of regulators as limiting cases. It was found that the value of the energy gap depends on the regulator parameters. We argue that the optimization based on the disappearance of the false, broken symmetric phase of the model leads to the Litim's regulator. The least sensitivity on the regulator parameters leads, however, to an IR regulator being somewhat different of the Litim's one, but it can be described as a perturbatively improved, or generalized Litim's regulator and provides analytic evolution equations, too.
\end{abstract}

Keywords: Renormalization group; critical exponents.

PACS numbers: 11.10.Gh, 11.10.Hi, 05.10.Cc

\section{Introduction}

The application of the functional renormalization group $(R G) \operatorname{method}^{1-6}$ for the quantized one-dimensional anharmonic oscillator is a highly nontrivial task. ${ }^{7-9}$ In the numerical treatment of the RG the problem arises from the fact that the ultraviolet (UV) double-well potential cannot become convex in the infrared (IR) regime, if the coupling of the anharmonic term is weak. The potential of the classical model, i.e. the potential at the UV scale can be either a simple convex potential or a doublewell potential with nontrivial minima. In the second case, the classical model has ground states with spontaneously broken $Z_{2}$ symmetry. In quantum mechanics, however, the effective potential should be convex due to the tunneling effect even if the RG evolution is started from the double-well UV potential; so the quantized model should have a single symmetric phase. 
Usually the energy gap between the ground state and the first excited state is the observable calculated for the anharmonic oscillator. The model can be also handled by solving the Schrödinger equation for the anharmonic potential numerically. Let us call the results of the latter exact. The exact results can then serve as guidelines in controlling and optimizing the results of the RG calculations.

The original optimization strategy is based on determining the IR regulator which provides the fastest convergence for the RG flows. ${ }^{10,11}$ A plausible optimization condition could be to find the IR regulator which gives the closest to the exact value of the observable. The problem of this reasoning is that, on the one hand, we have numerical results in RG with strong truncations in the gradient expansion, and the Taylor expansions in its functionals. On the other hand, the regulators can be deformed easily to provide a wide range of results which may reproduce any exact values for an observable. Therefore, we should choose another strategy for the optimization of the IR regulator. In this paper, we follow the optimization strategy which is based on choosing that value of the observable as the optimized one that shows the slightest dependence on the parameters of the regulator. Recently, this optimization strategy has often been used. ${ }^{12-18}$ At first glance, it seems to be rather a mathematical condition. Nevertheless, it can be made plausible by simple physical reasoning. The original generating functional does not contain the IR regulator, therefore it is reasonable to look for such physical results which have the least dependence on the regulator. However, the systematic search for the extremal value of any observable could not be performed among the various available IR regulators due to their rather different functional forms. This situation has been changed as the compactly supported smooth (css) IR regulator function has been introduced ${ }^{19}$ inspired by the so-called Salamon-Vertse potential used in nuclear physics. ${ }^{20-23}$ One can recast the css regulator into a simpler form that enables one to deform it continuously into the Litim's, the exponential and the power-law regulators by using only two parameters, ${ }^{24}$ and to perform the optimization program on a simple two-dimensional surface. By this technique we found successfully the least sensitive extremal value of the critical exponent $\nu$ of the correlation length for the quantum Einstein gravity and for the three-dimensional $O(1)$ model. ${ }^{24}$ Then, this method was also used successfully to investigate sine-Gordon type models. ${ }^{25}$ In the present paper, the same approach is used for the determination of the energy gap of the one-dimensional anharmonic oscillator.

We can find an optimized regulator different of the Litim's optimized one. However, the obtained optimized css regulator is very close to the Litim's result, so that one can consider it the perturbative generalization of the Litim's regulator. For the resulting new regulator one can perform the momentum integration in the $\mathrm{RG}$ equation in order to obtain for it a closed analytic form and that makes much more simple to handle the RG equation numerically.

The paper is organized as follows. In Sec. 2, the investigated model, the RG method, and the regulators are introduced. In Sec. 3, we discuss the truncations 
applied during the numerical calculations. We collect the results for the optimization strategies in Sec. 4. Finally, in Sec. 5 the conclusions are drawn up.

\section{Evolution Equations}

The RG method provides us a partial integro-differential equation for the effective action, which is called the Wetterich equation ${ }^{1,2}$

$$
\dot{\Gamma}_{k}=\frac{1}{2} \operatorname{Tr} \frac{\dot{R}_{k}}{R_{k}+\Gamma_{k}^{\prime \prime}},
$$

where ${ }^{\cdot}=k \partial_{k},{ }^{\prime}=\partial / \partial \phi, R_{k}$ is the regulator and the trace $\operatorname{Tr}$ denotes the integration over all momenta and summation for internal indices. Equation (1) has been solved over the functional subspace defined by the ansatz

$$
\Gamma_{k}=\int_{x}\left[\frac{Z_{k}}{2}\left(\partial_{\mu} \phi\right)^{2}+V_{k}\right],
$$

with the potential $V_{k}$, and the wave function renormalization $Z_{k}$. In the case of the local potential approximation (LPA) $Z_{k}=1$. Quantum mechanics can be considered as a quantum field theory with zero spatial and one time dimension, therefore one can apply the RG technique there, the field variable $\phi$ represents the oscillator coordinate. Then the evolution equation for the potential reads as

$$
\dot{V}_{k}=\frac{1}{2 \pi} \int_{0}^{\infty} d p \frac{\dot{R}_{k}}{p^{2}+R_{k}+V_{k}^{\prime \prime}},
$$

where $p$ stands essentially for the frequency in that case. The initial condition for Eq. (1) is given by the explicit form of the microscopic effective action at the UV cutoff $k=\Lambda$. There are lots of examples in the literature for different types of regulator functions. Here we use the dimensionless form of the css regulator,

$$
r_{\mathrm{css}}=\frac{s_{1}}{\exp \left[s_{1} y^{b} /\left(1-s_{2} y^{b}\right)\right]-1} \theta\left(1-s_{2} y^{b}\right),
$$

with $y=p^{2} / k^{2}$ and $r=r(y)$ is the dimensionless regulator $r=R / p^{2}$, furthermore $b \geq 1$ and $s_{1}, s_{2}$ are positive parameters. Unfortunately, the momentum integral in the evolution equation (3) has no analytic form for this regulator. For the limiting cases of the css regulator one recovers the following commonly used regulator functions, ${ }^{24}$

$$
\begin{aligned}
\lim _{s_{1} \rightarrow 0} r_{\mathrm{css}} & =\left(\frac{1}{y^{b}}-s_{2}\right) \theta\left(1-s_{2} y^{b}\right), \\
\lim _{s_{1} \rightarrow 0, s_{2} \rightarrow 0} r_{\mathrm{css}} & =\frac{1}{y^{b}} \\
\lim _{s_{2} \rightarrow 0} r_{\mathrm{css}} & =\frac{s_{1}}{\exp \left[s_{1} y^{b}\right]-1},
\end{aligned}
$$


where the first limit gives the Litim's regulator for $s_{2}=1$, the second one is the power-law regulator, and the third one gives the exponential regulator, if $s_{1}=1$. One can perform the optimization by finding an extremum of the energy gap on the parameter space spanned by $s_{1}$ and $s_{2}$. We note that the case $b=1$ satisfies the normalization conditions ${ }^{26}$

$$
\lim _{y \rightarrow 0} \mathrm{yr}=1 \quad \text { and } \quad \lim _{y \rightarrow \infty} \mathrm{yr}=0 .
$$

The usage of the power-law regulator with $b=1$ is usually called Callan-Symanzik (CS) scheme. We investigate the quantum mechanical anharmonic oscillator in terms of the Taylor-expanded potential

$$
V_{k}=\frac{m_{k}^{2}}{2} \phi^{2}+g_{k} \phi^{4}+\sum_{n=3}^{N} \frac{g_{2 n}(k)}{(2 n) !} \phi^{2 n},
$$

where besides the harmonic and the quartic anharmonic terms we have introduced the additional couplings $g_{2 n}$ with $n \geq 3$ which are generated by the RG method. Inserting Eq. (9) into Eq. (3) we obtain a system of ordinary differential equations for the couplings, as usual. The evolution equations for the couplings $m_{k}$ and $g_{k}$ are:

$$
\begin{aligned}
& \dot{m}_{k}^{2}=-\frac{12}{\pi} \int_{0}^{\infty} d p \dot{R}_{k} \frac{g_{k}}{\left(p^{2}+R_{k}+m_{k}^{2}\right)^{2}}, \\
& \dot{g}_{k}=\frac{1}{48 \pi} \int_{0}^{\infty} d p \dot{R}_{k}\left[\frac{3456 g_{k}^{2}}{\left(p^{2}+R_{k}+m_{k}^{2}\right)^{3}}-\frac{g_{6}}{\left(p^{2}+R_{k}+m_{k}^{2}\right)^{2}}\right] .
\end{aligned}
$$

The evolution equations for the further couplings have similar qualitative structures.

The solution of the RG equations in LPA provides us the effective potential $V_{0}$, i.e. the potential in the limit $k \rightarrow 0$. We look for the energy gap of the model given by

$$
\Delta E=\left.\sqrt{V_{0}^{\prime \prime}}\right|_{\phi=\langle\phi\rangle}
$$

where $\langle\phi\rangle$ is the vacuum expectation value of the field variable. In quantum mechanics the vacuum expectation value is the trivial field configuration, i.e. $\langle\phi\rangle=0$. From Eq. (9), the energy gap is

$$
\Delta E=m_{0}
$$

which is the IR limit of the coupling $m_{k}$.

\section{Truncations}

Performing the RG analysis of the quantized one-dimensional anharmonic oscillator we have used two kinds of truncations, that of the gradient expansion in its lowest order, the LPA, and that of the Taylor expansion of the local potential. We have restricted ourselves to the LPA because the field-dependence of the wave function renormalization cannot be handled by Taylor expansion due to its strange functional 


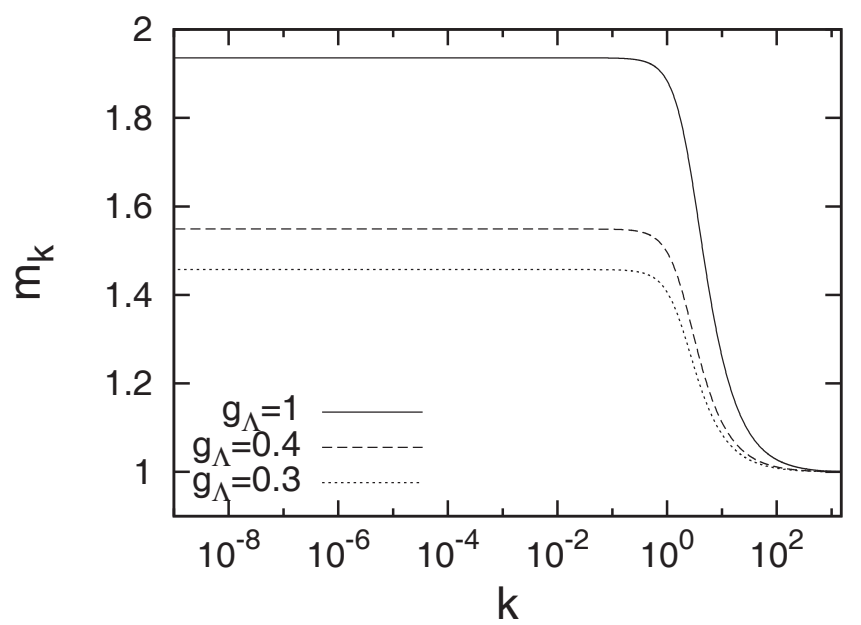

Fig. 1. The evolution of the coupling $m_{k}$ is shown for Callan-Symanzik scheme, for $m_{\Lambda}^{2}=1$. The curves correspond to various initial values of $g_{\Lambda}$.

form. ${ }^{8}$ At the UV scale chosen for $\Lambda=1500$ we set the couplings $m_{\Lambda}^{2}$ and $g_{\Lambda}$ and the further couplings are suppressed. We investigate the energy gap $\Delta E$ as the function of these initial values.

Figure 1 shows the flow of the coupling $m_{k}$ during the evolution in CS scheme for various initial values of the quartic coupling $g_{\Lambda}$. In the IR limit the dimensionful coupling $m_{k}^{2}$ as well as the other dimensionful couplings scale marginally, i.e. they tend to positive constant values.

The obtained numerical value of the energy gap is sensitive to many parameters in the calculations. Ideally, one should optimize the values of the energy gap for the regulator parameters $b, s_{1}, s_{2}$, and for $N$, i.e. the order of the expansion in Eq. (9). Throughout the present work, we set $b=1$ because only this choice satisfies the normalization condition in Eq. (8) for the regulator. We note, on the one hand, that previous results in the literature showed that the optimal value is around $b \approx 2$ in the two-dimensional sine-Gordon model $^{27}$ and in the three-dimensional $O(N)$ model. On the other hand, it is impossible to find an optimal value for the energy gap of the one-dimensional anharmonic oscillator by varying the value of the parameter $b$, because for various initial values of $m_{\Lambda}^{2}$ and $g_{\Lambda}$ one obtains various "optimal" values in the interval $b \in(1 \cdots 6)$.

The power-law regulator was chosen to explore the $N$-dependence, the results are demonstrated in Fig. 2. We choose the case $m_{\Lambda}^{2}<0$ for the optimization. Although one expects that larger values of $N$ could improve the approximation of the expansion, one can see in Fig. 2 that the numerical errors increase for too large $N$ values. Thus, one concludes that the optimal value of the number of couplings is about 6 in the LPA for the power-law regulator.

The results for the most important regulators are collected in Table 1. 


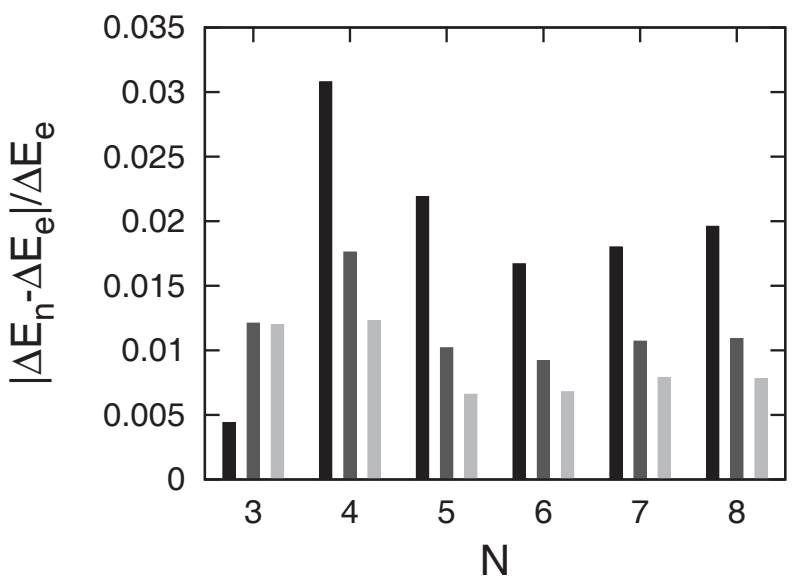

Fig. 2. The relative deviation of the numerical values of the energy gap $\left(\Delta E_{n}\right)$ from the exact ones $\left(\Delta E_{e}\right)$ as the function of $N$ is shown for various initial values of $g_{\Lambda}$ for $m_{\Lambda}^{2}=-1$. The black column corresponds to $g_{\Lambda}=0.4$, the dark grey column denotes $g_{\Lambda}=0.3$ and the light grey one refers to $g_{\Lambda}=0.2$. The data were calculated in the CS scheme.

Table 1. The value of the first energy gap is shown for various initial conditions. In the order of the columns it is shown the well-known exact values, the values calculated by Heat Kernel renormalization and the values calculated by us in the Litim, CS and exponential schemes.

\begin{tabular}{rllllll}
\hline$m_{\Lambda}^{2}$ & $g_{\Lambda}$ & $\Delta E_{\text {exact }}$ & $\Delta E_{\mathrm{HK}}$ & $\Delta E_{\text {Litim }}$ & $\Delta E_{\mathrm{CS}}$ & $\Delta E_{\text {exp }}$ \\
\hline 1 & 1 & 1.9341 & 1.9380 & 1.9386 & 1.9358 & 1.9382 \\
1 & 0.4 & 1.5482 & 1.5498 & 1.5507 & 1.5490 & 1.5504 \\
1 & 0.1 & 1.2104 & 1.2109 & 1.2110 & 1.2105 & 1.2109 \\
1 & 0.05 & 1.1208 & 1.1210 & 1.1211 & 1.1208 & 1.1210 \\
1 & 0.03 & 1.0779 & 1.0780 & 1.0780 & 1.0779 & 1.0778 \\
1 & 0.02 & 1.0540 & 1.0542 & 1.0542 & 1.0541 & 1.0542 \\
-1 & 0.4 & 0.9667 & 0.9730 & 0.9778 & 0.9733 & 0.9772 \\
-1 & 0.3 & 0.8166 & 0.8233 & 0.8288 & 0.8241 & 0.8281 \\
-1 & 0.2 & 0.6159 & 0.6227 & 0.6309 & 0.6262 & 0.6302 \\
\hline
\end{tabular}

For negative values of $m_{\Lambda}^{2}$ and for small values of $g_{\Lambda}$ the RG approach does not work, the effective potential becomes concave at $\phi=0$ and we have no result for the energy gap. This happens presumably due to the strong truncation of the potential and that of the gradient expansion. Table 1 shows that the choice $N=6$ provides rather close values of the energy gap for the various kinds of regulators. Their deviations from each other are much less then their deviations of $\sim 1 \%$ from the exact values for $m_{\Lambda}^{2}>0$. Similar is true when $m_{\Lambda}^{2}<0$ and when $g_{\Lambda}$ is sufficiently large. 


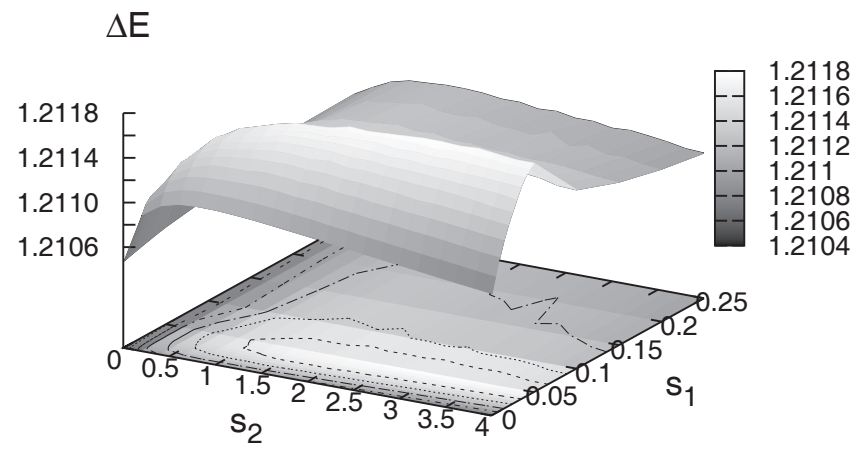

Fig. 3. The energy gap $\Delta E$ is shown as the function of the regulator parameters $s_{1}$ and $s_{2}$. The initial couplings are $m_{\Lambda}^{2}=1$ and $g_{\Lambda}=0.1$. We set $b=1$.

\section{Optimization Strategies}

For the further investigations, we set $N=6$ and look for the extremum of the energy gap in the parameters $s_{1}$ and $s_{2}$. In Fig. 3 , we plotted $\Delta E$ for different regulator parameters and for positive $m_{\Lambda}^{2}$. Interestingly, the results show very slight regulator-dependence. We had to go beyond 3 digits in the numerical precision to find some nontrivial results. As a comparison we note that in the case of the optimization of the model of quantum Einstein gravity ${ }^{24}$ we obtained very strong regulator-dependence. There the value of the critical exponent could change several orders of magnitude, and even its sign could change. The anharmonic oscillator investigated here is a one-dimensional model and this can be the reason of such a weak regulator-dependence. The removal of UV divergences in higher-dimensional models may introduce strong scheme-dependence, while there is no need to remove UV divergences in one-dimensional models. Furthermore there is no IR singularity due to the positive mass term.

It would be the most straightforward optimization strategy to recover the exact, i.e. physical value of the observable $\Delta E$ for a given IR regulator. Figure 3 shows for a particular choice of the initial conditions, that the exact value of the energy gap $\Delta E=1.2104$ can be obtained by the power-law regulator near the origin $s_{1} \approx 0$ and $s_{2} \approx 0$ of the parameter space. Unfortunately, other initial conditions require other IR regulators with different regulator parameters. Although this strategy can be supported mostly by physical arguments and it can be the only reasonable optimization, nevertheless it does not work in our RG framework. This strategy may work when the truncations are minimal, which is not the case in our treatment.

It is another possibility for the optimization to look for that value of the observable $\Delta E$ which shows the least sensitivity to the regulator parameters, i.e. to find an extremum of the energy gap in the parameter space. At the maximum of the surface in Fig. 3 the sensitivity of $\Delta E$ is minimal to $s_{1}$ and $s_{2}$. Figure 3 shows that there is a maximum of the energy gap at $s_{1}=0.05$ and $s_{2}=3$. Accordingly, the 


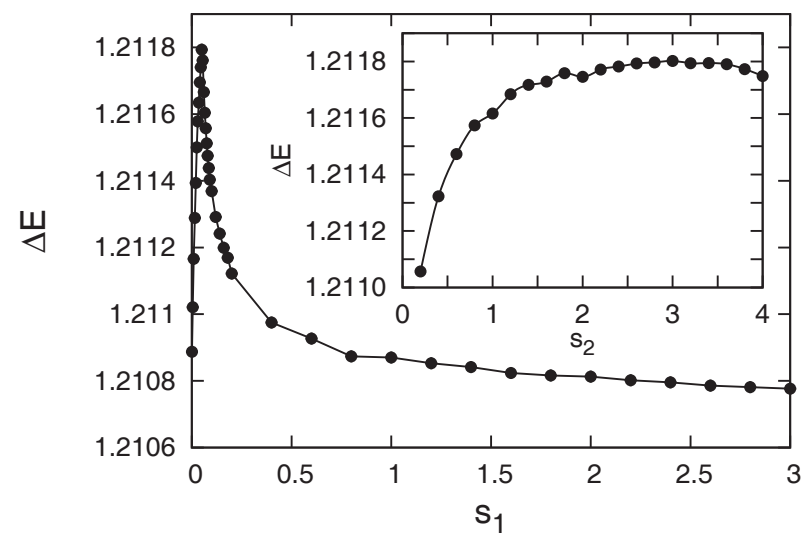

Fig. 4. The sections of the surface plot are shown through the extremum. The initial couplings are $m_{\Lambda}^{2}=1$ and $g_{\Lambda}=0.05$. We set $s_{2}=3$ and $s_{1}=0.05$ in the inset.

optimized regulator corresponds to the css regulator of the form

$$
r_{\mathrm{opt}}=\frac{0.05}{\exp [0.05 y /(1-3 y)]-1} \theta(1-3 y) .
$$

For other initial couplings we have got by means of this least-sensitivity optimization the same results with the same optimized IR regulator. The value of $s_{1}=0$ corresponds to the limit of a general Litim's regulator in Eq. (5). Figure 3 shows that this Litim's regulator zone gives neither the minimal value nor an extremum for $\Delta E$. In Fig. 4, we demonstrate how the energy gap changes close to the Litim's limit. There is a maximum at $s_{1}=0.05$ for practically all of the sections with $s_{2}=$ const. These maxima create a saddle along the $s_{2}$ direction for the small values $s_{1} \approx 0.05$. The $s_{1}=0.05$ section of the saddle is plotted in the inset of Fig. 4 . This curve also has a local maximum at $s_{2}=3$.

The minimal sensitivity optimization works well if we choose a positive initial value $m_{\Lambda}^{2}$ when both the blocked potential and the resulting effective potential are convex. However, for negative initial values $m_{\Lambda}^{2}$ the convexity cannot be granted. The quantized anharmonic oscillator can have only a symmetric phase, the spontaneously broken symmetric phase is excluded by the tunneling effect. For sufficiently small initial values of $g_{\Lambda}$ there is no room to turn the concave blocked potential into a convex one during the RG evolution. This appears probably due to the strongly truncated gradient expansion of the effective action. ${ }^{7,8}$ Taking into account the wave function renormalization and solving the RG equations without Taylor expansion may improve the treatment, i.e. may enable one to determine $\Delta E$ even for smaller initial values of $g_{\Lambda}$.

The determination of the smallest initial value of $g_{\Lambda}$ for which the effective potential becomes convex gives another possibility to optimize the IR regulator, since the model should have only a symmetric phase. We note that the disappearance of the false phase has been used recently to find optimized IR regulators for 
Table 2. The energy gap is shown as the function of the regulator parameters $s_{1}$ and $s_{2}$ for the initial couplings $m_{\Lambda}^{2}=-1$ and $g_{\Lambda}=0.08$.

\begin{tabular}{lcccc}
\hline & $s_{2}=0.001$ & $s_{2}=1$ & $s_{2}=2$ & $s_{2}=3$ \\
\hline$s_{1}=0.001$ & - & 0.23538 & 0.23679 & 753913.25671 \\
$s_{1}=0.05$ & - & 0.23598 & 0.23785 & 23102.53408 \\
$s_{1}=1$ & 0.23766 & 0.23556 & 341061.15077 & - \\
$s_{1}=2$ & 0.23612 & 0.23604 & - & - \\
$s_{1}=3$ & 0.23566 & 0.23644 & - & - \\
\hline
\end{tabular}

sine-Gordon type models. ${ }^{28}$ We found that $g_{\Lambda}=0.08$ is the smallest initial value for which the energy gap can be determined reliably in the RG framework used by us. We note that in Ref. 8 the smallest value is $g_{\Lambda}=0.02$ which is a better result. Here we cannot have such a precision, because we did not include the wave function renormalization and Taylor-expanded potential that was avoided in Ref. 8. Nevertheless, the issue of optimization is important even if the RG framework involves quite strong truncations, like in our case. In Table 2, we collected the results for the energy gap for $m_{\Lambda}^{2}=-1$ and $g_{\Lambda}=0.08$.

The extremely large values show numerical instabilities during the calculations. Table 2 shows that the IR regulator in Eq. (13) is not the optimized one in Eq. (13). If one defines the optimized regulator via finding the smallest $g_{\Lambda}$ which restores the convexity of the potential, then the Litim's regulator proves to be the best one, since it gives the smallest value of $\Delta E$ there. It seems that various optimization procedures give different IR regulators.

The IR regulator Eq. (13) is very close to the Litim's one. If one Taylor expands the css regulator in $s_{1}$ at $s_{1}=0$ then one obtains that

$$
r_{\text {pert }} \approx\left(\frac{1-s_{2} y^{b}}{y^{b}}-\frac{s_{1}}{2}+\frac{y^{b} s_{1}^{2}}{12\left(1-s_{2} y^{b}\right)}+\cdots\right) \theta\left(1-s_{2} y^{b}\right) .
$$

Up to the linear term in $s_{1}$ the LPA evolution equation for the potential takes the form

$$
\dot{V}=\frac{1}{2 \pi} \int_{0}^{\frac{k}{\sqrt{s_{2}}}} d p \frac{2 k^{2}}{k^{2}+p^{2}\left(1-s_{2}-\frac{s_{1}}{2}\right)+V^{\prime \prime}} .
$$

The perturbative Litim's regulator in Eq. (14) does not cancel the momentum dependence in the integrand of the loop integral. In this sense the regulator $r_{\text {pert }}$ takes after the CS type regulator, however the UV divergence does not appear, since the upper integration limit is restricted by the $\theta$ function and it guarantees the finiteness in any dimensions. Moreover, the resulting $\mathrm{RG}$ equation remains analytic. In $d=1$, it reads as

$$
\dot{V}=\frac{k^{2}}{\pi} \frac{1}{\sqrt{\left(1-s_{2}-\frac{s_{1}}{2}\right)\left(k^{2}+V^{\prime \prime}\right)}} \tan ^{-1}\left(\sqrt{\frac{\left(1-s_{2}-\frac{s_{1}}{2}\right) k^{2}}{s_{2}\left(k^{2}+V^{\prime \prime}\right)}}\right) .
$$




\section{Conclusions}

By using the functional RG method we calculated the energy gap for the quantized one-dimensional anharmonic oscillator. The RG approach requires approximations which can introduce some regulator-dependence. We used the LPA and the Taylor expansion of the potential with a truncation yielding the smallest deviation of the energy gap of the oscillator from its exact value. The regulator-dependence of the results has been investigated by making use of the css regulator that enables one to consider various types of regulator functions in a unique parametrization. The css regulator Eq. (4) depends on the parameters $b, s_{1}$ and $s_{2}$. We set $b=1$ for our study when the normalization conditions Eq. (8) are satisfied. The optimization of the css regulator with respect to the parameters $s_{1}$ and $s_{2}$ has been carried out.

For the anharmonic oscillator with a single-well UV potential, it turned out that the optimization strategy based on the minimal sensitivity on the regulator parameters works rather well. It is found that the energy gap has an extremum as the function of the regulator parameters. The optimized regulator found in that manner is shown to be the generalization of the Litim's regulator and it provides an analytic evolution equation for the potential in $d=1$. For the anharmonic oscillator with a double-well UV potential, this generalized Litim's regulator seems not to be the optimal one. Instead of the optimization via achieving the minimal sensitivity of the observable on the regulator parameters another optimization strategy can be followed. Then one looks for the regulator that enables one to reestablish the convexity of the numerically determined effective potential for the smallest value of the quartic coupling. We have found that the Litim's regulator appears in that case rather optimal instead of the generalized Litim's regulator introduced in the case of the single-well potential. It is argued that such a situation is due to the strong truncations in the gradient expansion and in the Taylor expansion of the potential.

\section{Acknowledgments}

This research was supported by the European Union and the State of Hungary, cofinanced by the European Social Fund in the framework of the TÁMOP 4.2.4.A/211-1-2012-0001 'National Excellence Program'. Sándor Nagy acknowledges financial support from a János Bolyai Grant of the Hungarian Academy of Sciences and the Hungarian National Research Fund OTKA (K112233).

\section{References}

1. C. Wetterich, Phys. Lett. B 301, 90 (1993).

2. J. Berges, N. Tetradis and C. Wetterich, Phys. Rep. 363, 223 (2002).

3. J. Polonyi, Central Eur. J. Phys. 1, 1 (2003).

4. J. M. Pawlowski, Ann. Phys. 322, 2831 (2007).

5. H. Gies, Lect. Notes Phys. 852, 287 (2012).

6. O. J. Rosten, Phys. Rep. 511, 177 (2012).

7. A. Kapoyannis and N. Tetradis, Phys. Lett. A 276, 225 (2000). 
8. D. Zappala, Phys. Lett. A 290, 35 (2001).

9. S. Nagy and K. Sailer, Ann. Phys. 326, 1839 (2011).

10. D. F. Litim, Phys. Lett. B 486, 92 (2000).

11. D. F. Litim, Phys. Rev. D 64, 105007 (2001).

12. L. Canet, B. Delamotte, D. Mouhanna and J. Vidal, Phys. Rev. D 67, 065004 (2003).

13. L. Canet, B. Delamotte, D. Mouhanna and J. Vidal, Phys. Rev. B 68, 064421 (2003).

14. K. Essafi, J. Kownacki and D. Mouhanna, Europhys. Lett. 98, 51002 (2012).

15. M.-F. Li and M. Luo, Phys. Rev. D 85, 085027 (2012).

16. M.-F. Li and M. Luo, Phys. Rev. D 88, 085019 (2013).

17. K. Essafi, J. P. Kownacki and D. Mouhanna, Phys. Rev. E 89, 042101 (2014).

18. T. Kloss, L. Canet, B. Delamotte and N. Wschebor, Phys. Rev. E 89, 022108 (2014).

19. I. Nandori, J. High Energy Phys. 1304, 150 (2013).

20. P. Salamon and T. Vertse, Phys. Rev. C 77, 037302 (2008).

21. P. Salamon, A. Kruppa and T. Vertse, Phys. Rev. C 81, 064322 (2010).

22. A. Racz, P. Salamon and T. Vertse, Phys. Rev. C 84, 037602 (2011).

23. P. Salamon, R. G. Lovas, R. M. Id Betan, T. Vertse and L. Balkay, Phys. Rev. C 89, 054609 (2014).

24. S. Nagy, B. Fazekas, L. Juhasz and K. Sailer, Phys. Rev. D 88, 116010 (2013).

25. I. Marian, U. Jentschura and I. Nandori, J. Phys. G 41, 055001 (2014).

26. M. Reuter and F. Saueressig, Phys. Rev. D 65, 065016 (2002).

27. I. Nandori, Phys. Rev. D 84, 065024 (2011).

28. I. Nandori, I. Marian and V. Bacso, Phys. Rev. D 89, 047701 (2014). 